\title{
NOUVELLES RECOLTES DE CHIRONOMIDES EN CAMARGUE ET DANS LES MARISMAS DU GUADALQUIVIR
}

\author{
[Diptères ]
}

par H. Laville et J.-N. Toureng.

Dans une note préliminaire [Toureng 1966], l'un de nous avait signalé la récolte de 29 espèces de Chironomides en Camargue. Les recherches faunistiques poursuivies pendant ces deux dernières années nous permettent d'augmenter cette liste des noms de 17 espèces dont deux seulement avaient déjà été capturées sur le littoral camarguais par REMMERT [1953] = Thalassomyia frauenfeldi, espèce de la sous-famille typiquement marine des Clunioninae, et Chironomus salinarius dont la larve colonise également les milieux salés. Cet auteur avait également signalé en Camargue et sur le littoral de la Côte Vermeille la présence de Chironomus halophilus, Chironomus annularius, Polypedilum nubeculosum et Cricotopus vitripennis.

Des chasses très fréquentes tout au long de l'annéc nous permettent de donner, pour chacune des 46 espèces récoltées jusqu'à ce jour, les périodes de vol des imagos ainsi que quelques remarques d'ordre systématique, biogéographique, écologique ou biologique pour les espèces nouvellement citées. Les stations de référence correspondent à celles décrites dans la note préliminaire.

Une première prospection effectuée lors d'un bref séjour aux Marismas (3 avril-17 mai 1965) nous avait laissé entrevoir la similitude de la Faune Chironomidienne dans les deltas du Rhône et du Guadalquivir et nous avait fourni le matériel nécessaire pour l'étude systématique de trois Chironomides présents à la fois dans ces deux régions [Laville-Toureng 1967]. Au cours de plusieurs voyages en Andalousie, H. HAfNer a eu la gentillesse de récolter à notre intention un abondant matériel dans lequel, sur les 24 espèces dénombrées, 20 sont communes aux deux plaines deltaïques. Les prélèvements, effectués au filet entomologique, de janvier 1966 à octobre 1967, nous permettent de donner pour chaque espèce les périodes de vol des imagos. 
I. - Liste complémentaire à l'inventaire faunistique des Chironomides de Camargue ${ }^{1}$.

\section{TANYPODINAE}

Ablabesmyia monilis (L.)

Espèce à vaste répartition européenne. Les larves s'accommodent des eaux légèrement saumâtres du marais de Saint-Seren (station IV).

Procladius culiciformis (MG.)

Largement répartie en Europe, cette espèce a été abondamment récoltée en mars près des mares temporaires d'eau douce des Cerisières, du Clos des Faïsses, de l'Esquineau, le plus souvent associée à Psectrocladius edwardsi et Micropsectra foliata. Des élevages ont confirmé l'origine de ces espèces.

\section{ORTHOCLADIINAE}

Cricotopus trifasciatus (PAnz.)

Connue dans toute l'Europe (les larves de cette espèce sont mineuses de Potamogeton natans (KeTtisch 1937) qu'on rencontre en Camargue principalement aux stations II, III et V).

*Cricotopus pallidipes Edw.

Connue uniquement d'Angleterre (EDwards 1929), cette espèce a été le plus souvent capturée près des rizières ou le long de leurs canaux d'irrigation, de mai à septembre.

Cricotopus stagnorum (G.) ${ }^{2}$

Signalée de Fos-sur-Mer, à l'est de l'embouchure du Grand Rhône et de l'îlot de Planier, au large de Marseille (sous le nom de Trichocladius maritimus) [Goetghebuer 1940-1950]. Des éclosions ont été obtenues à partir de larves prélevées dans des milieux sursalés pouvant atteindre des concentrations en $\mathrm{NaCl}$ de 67 à $125 \mathrm{~g} / \mathrm{l}$.

Camptocladius stercorarius d. GEER.

Espèce terrestre, connue de presque toute l'Europe.

Pseudosmittia trilobata (EDw.)

Espèce également terrestre assez largement répandue en Europe.

1. Les noms d'espèces précédés d'un astérisque $\left(^{(*)}\right.$ sont nouveaux pour la Faune de France.

2. C. maritimus est considérée par Hinvenoja comme synonyme de $C$. stagnorum, elle-mếme probablement synonyme de Trichocladius seurati décrite par Goetghebuer des flaques supralittorales de l'Algérie (in litt.). 


\section{Clunioninae}

Thalassomyia frauenfeldi (ScHIN.)

Répartition [Remment 1956].

Récoltée d'avril à juin, le long de la côte qui s'étend entre les deux bras du Rhône. Les larves de cette espèce vivent dans les algues recouvrant les rochers sans cesse éclaboussés par les embruns marins.

\section{Chironominae}

Camptochironomus pallidivittatus MALI.

Très souvent associée à Camptochironomus tentans à la station IV en mai et en juin, période pendant laquelle la salinité atteint son maximum (de $4 \mathrm{~g} / \mathrm{l}$ à $12 \mathrm{~g} / \mathrm{l}$ ).

Chironomus dorsalis (MG.)

De répartition holarctique, cette espèce a pu être récoltée aux mêmes périodes que Chironomus gr. thummi (février à mai).

*Chironomus luridus STR.

Moins précoce que l'espèce précédente puisqu'elle n'apparaît qu'en avril aux stations IV et VI et éclot jusqu'en juillet près des canaux d'irrigation.

\section{Chironomus salinarius $\mathbf{K}$.}

Cette espèce signalée par Remmert [1953] dans le Vaccarès, l'Etang de Salses et l'Étang de Canet, a été rencontrée, le plus souvent associée à Cricotopus maritimus et à Cricotopus vitripennis var. halophilus, dans les bassins de concentration de sels de la S.A.L.I.C.A.M.

\section{Chironomus thummi thummi STR.}

Des exemplaires aux métanotums semblables à ceux figurés par Moeller [1966] coexistent avec ceux de la sous-espèce piger et présentent les mêmes périodes de vol. Ces deux sous-espèces ont été retrouvées en Espagne avec, toutefois, des périodes de vol différentes.

Glyptotendipes barbipes (STAEg.) = Glyptotendipes sp. [ToURENQ 1966 ].

La larve et la nymphe de cette espèce sont signalées pour la première fois en France par Govin [1936]. Des imagos $\hat{o}$ et $q$ ont été obtenus par élevage de larves prélevées aux stations I, II (eau douce), IV et VI (eau saumâtre). 
Harnischia virescens $\mathrm{K}$.

Des imagos ont pu être capturés à la lumière, toujours près des canaux d'irrigation des rizières.

Harnischia fuscimana $\mathrm{K}$.

Les spécimens de Camargue coïncident avec la description de KiEfFER reprise par GoETGHEBUER [1928]; toutefois seule la moitié distale de leur métanotum est brune, alors qu'il est signalé entièrement noir dans la description de Goetghebuer.

Lenzia flavipes MG.

Espèce à répartition holarctique récoltée en mai, septembre et novembre près du canal de "l'Aube de Bouic», comparable à la station II.

\section{Micropsectra foliata LAv.}

Les individus récoltés en Camargue sont de taille plus grande $(4,5-5,5 \mathrm{~mm})$ que ceux des Pyrénées $(3,5-4 \mathrm{~mm})$. Leur $\mathrm{AR}=1,6$ 1,8 est également plus élevé que celui de la population pyrénéenne $(\mathrm{AR}=1,17)$.

Cette espèce a été obtenue par élevage de larves prélevées dans les Mares des Cerisières.

\section{II. - Périodes de vol des Chironomides de Camargue (fig. 1).}

Le maximum d'éclosions se situe de mars à juillet, la plus grande diversité spécifique se rencontre en mai (41 espèces).

La diminution du nombre des espèces de Chironomides observée en août coïncide avec l'assèchement des marais et des mares temporaires.

En octobre, sur les 6 espèces récoltées, 4 (Cricotopus vitripennis var. halophilus, Cricotopus maritimus, Chironomus halophilus, Chironomus salinarius) proviennent des milieux salés qui restent en eatu tout l'été.

La remise en eau progressive des marais et des étangs, due aux précipitations de septembre et d'octobre, permet en novembre la réapparition des imagos de certaines espèces : Cricotopus silvestris var. ornatus, Psectrocladius edwardsi, Microtendipes pedellus.

22 espèces, c'est-à-dire presque la moitié des espèces recoltées ont une longue période de vol qui peut durer 5 mois ou plus.

III. - Relevé faunistique et périodes de vol des Chironomides des Marismas (fig. 2).

A l'exception d'Ablabesmyia monilis (sous le nom de Tanypus monilis) et de Cricotopus sylvestris récoltés dans la région de Bar- 


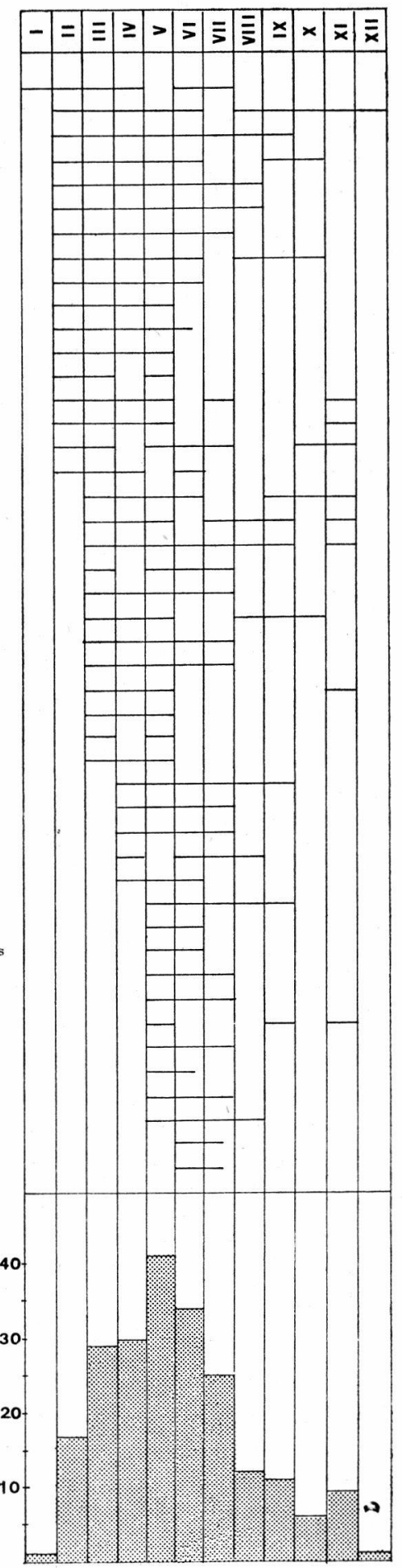

Fig. 1. - Périodes de vol des Chironomides de Camargue. Le diagramme représente le nombre total d'espèces capturées aux différents mois de l'année. 
celone [MargaleF 1944 a et b] et des trois espèces déja signalées d'Andalousie [LAviLle-Toureng 1967], toutes les espèces citées sont, à notre connaissance, nouvelles pour la faune d'Espagne.
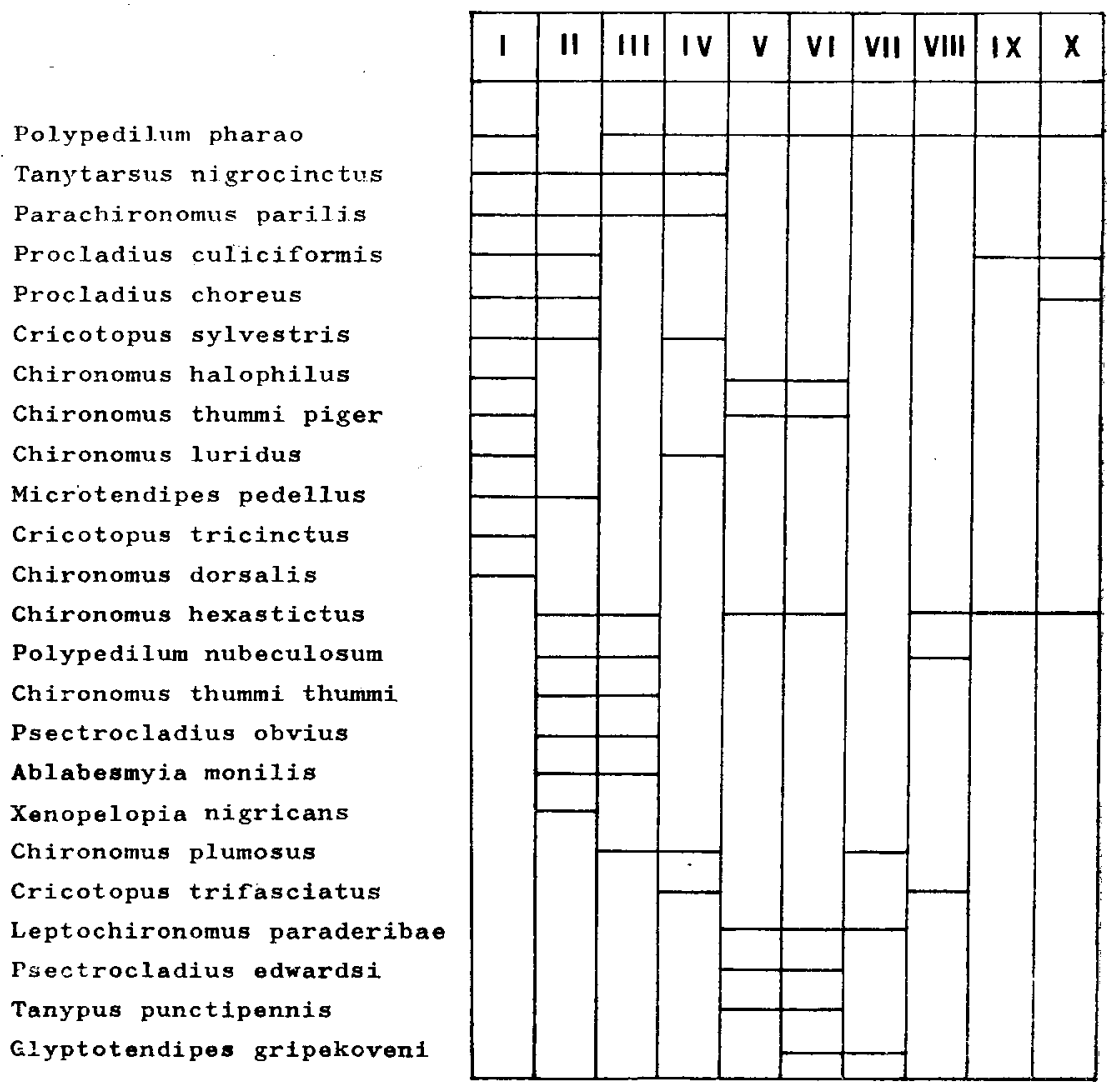

Fig. 2. -- Périodes de vol des Chironomides des Marismas.

Tanytarsus nigrocinctus $s^{3}$ est récoltée pour la première fois sur le continent européen. Cette espèce a été décrite par FrEEMAN [1957 a et b] d'Afrique équatoriale (Ouganda, Kenya, Congo Belge).

Dans le matériel de Camargue et des Marismas nous avons constaté la présence de 12 espèces signalées des îles de l'Atlantique au large des côtes marocaines ou portugaises dans les travaux de Santos Abreu [1918], Stor Å [1936] pour les Iles Canaries, Stor A [1949], Freemann [1957] pour l'Ile de Madère, Freemans [1957] enfin pour l'archipel des Açores : Ablabesmyia monilis, Procladius

3. Nous remercions le $\mathrm{D}^{\mathrm{r}}$ Reiss (Plön) pour la confirmation du nom de cette espèce. 
choreus, Cricotopus silvestris v. ornatus, Cricotopus vitripennis v. halophilus, Camptocladius stercorarius, Thalassomyia frauenfeldi, Camptochironomus tentans, Chironomus dorsalis, Chironomus plumosus, Chironomus gr. thummi, Limnochironomus nervosus, Polypedilum nubeculosum. Ce sont presque toutes des espèces holarctiques ou à large répartition européenne.

Des chasses moins intensives ainsi que l'exceptionnelle sécheresse de l'été 1967 expliquent, en partie, que le nombre d'espèces récoltées aux Marismas soit moins élevé qu'en Camargue; en effet, de juin à octobre les imagos ne purent être capturés qu'autour des «lucios», petites mares restant en eau alors que la majeure partie des marais andalous était à sec à cette période.

On peut également remarquer la précocité de la faune des Marismas puisque, dès le mois de février, $75 \%$ des espèces ont déjà fait leur apparition alors qu'à la même époque $30 \%$ seulement ont éclos en Camargue; on doit attendre le mois d'avril pour atteindre le même pourcentage d'éclosions qu'en Andalousie. Un tel décalage est la conséquence des conditions climatiques amenant un réchauffement plus rapide des eaux dans cette région méridionale de l'Espagne.

\section{RESUME}

Les auteurs donnent une liste de 17 espèces complémentaires à l'inventaire faunitique des Chironomides de Camargue ainsi que les périodes de vol, observées sur deux années, des 46 espèces qui y ont été récoltées jusqu'à ce jour. Ils donnent également un premier relevé faunistique comprenant 29 noms de Chironomides capturés en Andalousie, ainsi que leur période de vol. L'espèce Tanytarsus nigrocinclus est nouvelle pour le continent européen.

\section{NEW COLLECTIONS OF CHIRONOMIDS IN THE CAMARGUE AND IN THE MARISMAS OF THE GUADALQUIVIR}

The authors give a list of 17 species, complementary to the faunainventory of the Chironomids of the Camargue, as well as the periods of flight, observed over a space of two years for the 46 species which were collected there till now. They also give a first list of fauna, including 29 names of Chiromids found in Andalousia, as well as their period of flight. The species Tanytarsus nigrocinctus is new for the European continent.

\section{NEUE SAMMLUNGEN VON CHIRONOMHEN IN DER CAMARGUE UND IN DEN MARISMAS DES GUADALQUTVIRS}

Die Autoren geben eine zusätzliche Liste von 17 Arten, dic dic bekannte Fauna der Chironomiden aus der Camargue vervollständigt, sowie, beobachtet über zwei Jahre indurch, die Flugperioden der bisher bckannten 46 Arten. Ausserdem geben Sie erstmals ein Faunenver- 
zeichnis der 29 Chironomiden Arten die je in Andalusien gefangen wurden, sowie ihre Flugperiode. Die Art Tanytarsus nigrocinctus ist neu für den europäischen Kontinent.

\section{TRAVAUX CITÉS}

EDwards (F.W.). 1917. - British non biting midges (Dipt. Chiron.). Trans. R. Ent. Soc. London, 77 (2) : 279-430.

Freeman (P.). 1957 a. - Exploration hydrobiologique des lacs Kivu, Edouard et Albert (1952-1954). Inst. Royal Sc. Nat. Belgique (Bruxelles), 3 (2) : 205-221.

Freeman (P.). 1957 b. - A study of the Chironomidae of Africa. Part III. Bull. Brit. Mus., 5 (9) : 1-396.

Freeman (P.). 1959. - Chironomidae from the Azores and Madeira. Opusc. Ent., 24 (1-2) : 117-124.

Goetghebuer (M.) 1928. - Diptères Chironomidae. III. Chironomariac. In Faune de France, 18, Lechevalier éd. Paris : 1-174.

Goetghebuer (M.). 1940-1950. - Tendipedidae (Chironomidae.) f) Subfamilie Orthcladiinae. A. Die Imagines. In Lindner, Die Fliegen der paläarktischen Region, $13 \mathrm{~g}: 1-208$.

KetTisch (J.). 1937. - Zur Kenntnis der Morphologie und ökologie der Larve von Cricotopus trifasciatus. Konowia, 16 : 153-163.

Kieffer (J. J.). 1924-1925. - Chironomides d'Egypte. Bull. Soc. Ent. Egypte, 2-4 : 244-313.

Laville (H.) et TourenQ (J.-N.). 1967. - Contribution à la connaissance de trois Chironomides de Camargue et des Marismas du Guadalquivir. Annls Limnol., 3 (1) :185-204.

Margalef (R.). 1944 a. - Notas sobre Quironomidos. I. Graellsia 1. (2) : 3-13.

Margalef (R.). 1944 b. - - Notas sobre Quironomidos. II. Graellsia 3 (2) : 65-76.

MoELder (J.). 1966. - Die Färbung als diagnostisches Merkmal bei Chironomiden. Gewäs. Abwäss., 41/42 (Vehr. 1. Int. Symp. Chironomiden) : 38-43.

Remmert (H.). 1953. - Les Diptères des côtes méditerranéennes de France et d'Espagne.Vie et Milieu, 4 (3) : 540-546.

Remmert (H.). 1955. - Ökologische Untersuchungen über die Dipteren der Nord-und Ostsee. Arch. Hydrobiol., 51 (1) : 1-53.

Santos Abreu (E.). 1918. - Ensayo de una monografia de los Tendipedidos de las Islas Canarias. Mems R. Acad. Cienc. Artes Barcelona, 14 (2) : 159-325.

Sтон $\dot{A}$ (R.). 1936. - Chironomidae in Frey, Die Dipterenfauna der Kanarischen Inseln und ihre Probleme. Soc. Scient. Fenn. Comment. Biol., 6 (1) : 21-30.

STORA (R.). 1949. - Chironomidae in Frey, Die Dipterenfauna der Inseln Madeira. Soc. Scient. Fenn. Comment. Biol., 8 (16) : 15-16.

Tourene (J.-N.). 1966. - Introduction à l'étude écologique des Chironomides de Camargue. Annls Limnol., 2 (2) : 459-465.

(Laboratoire d'Hydrobiologie

de la Faculté des Sciences de Toulouse, Equipe de recherche associé au C.N.R.S.) 